\title{
Effects of Prickly Pear Dried Leaves, Artichoke Leaves, Turmeric and Garlic Extracts, and Their Combinations on Preventing Dyslipidemia in Rats
}

\author{
Nidal A. Qinna, ${ }^{1}$ Basma S. Kamona, ${ }^{1}$ Tawfiq M. Alhussainy, ${ }^{1}$ Hashem Taha, ${ }^{2}$ \\ Adnan A. Badwan, ${ }^{3}$ and Khalid Z. Matalka ${ }^{1}$ \\ ${ }^{1}$ Department of Pharmacology and Biomedical Sciences, Faculty of Pharmacy and Medical Sciences, Petra University, \\ Amman 11196, Jordan \\ ${ }^{2}$ Delass Natural Products, Naour 11710, Jordan \\ ${ }^{3}$ Jordanian Pharmaceutical Manufacturing Co. PLC. (JPM), Naour 11710, Jordan
}

Correspondence should be addressed to Nidal A. Qinna, nqinna@uop.edu.jo

Received 4 April 2012; Accepted 3 May 2012

Academic Editors: G. Biala, H. Cerecetto, G. Edwards, G. Froldi, G. Gervasini, and F. Komada

Copyright (C) 2012 Nidal A. Qinna et al. This is an open access article distributed under the Creative Commons Attribution License, which permits unrestricted use, distribution, and reproduction in any medium, provided the original work is properly cited.

\begin{abstract}
The successful use of herbal combinations in managing diseases or conditions over a single herb has lead us to evaluate the antidyslipidemic properties of the combination of the artichoke leaves extract, turmeric extract, prickly pear dried leaves (PPL) and garlic extract versus each one alone in two different hyperlipidemic animal models. A two-week treatment of each of the natural extracts, combination 1 (artichoke, turmeric and PPL) or combination 2 (artichoke, turmeric, PPL and garlic) prior to a single intraperitoneal injection of Pluronic F-127 resulted in decreasing significantly serum LDL levels by garlic and PPL extracts and serum LDL/HDL ratios by turmeric, PPL, combination 1 and 2. In a 10-day high fat diet model, only the combination 1 and 2 lowered serum cholesterol, LDL by $8-12 \%$, decreased significantly triglycerides, LDL/HDL ratio; and increased significantly HDL $(P<0.0001)$. However, a long term treatment of each natural product for 7 weeks resulted in decreasing significantly serum LDL levels and LDL/HDL ratio $(P<0.05-0.0001)$. Furthermore, only artichoke and PPL inhibited significantly HMG-CoA reductase activity $(P<0.05)$. In conclusion, short term, as well as long term, treatment using the combination of artichoke, turmeric, PPL and garlic extract prevents dyslipidemia; partially through inhibiting HMG-CoA reductase.
\end{abstract}

\section{Introduction}

Dyslipidemia can be defined as the elevation of cholesterol, triglycerides, and low-density lipoprotein (LDL) cholesterol serum levels while maintaining low serum levels of highdensity lipoprotein (HDL) cholesterol. This imbalance is considered a high-risk factor for inducing atherosclerosis and cardiovascular diseases (CVD). CVD is now considered the most common cause of death in both western and eastern countries [1].

Traditionally, natural remedies were used to overcome such imbalance in lipids metabolism and are claimed to be useful in controlling hyperlipidemia and associated pathologies [2]. Plants such as artichoke (Cynara scolymus) is an important component of the Mediterranean diet and it is rich in bioactive phenolic compounds, inulin, fibers, and minerals [3]. Traditionally, artichoke leaves were used for a variety of diseases [4]. It has been used to treat dyspepsia mainly because of its choleretic effect that is associated with increased bile formation [5]. In addition, artichoke extract has been found to exhibit antioxidant properties in cultured endothelial cells and monocytes [4] and has lipid lowering properties [3, 6-8].

Curcuma longa, commonly known as turmeric, is a rhizomatous perennial plant from the family Zingiberaceae $[9,10]$. Curcumin has been identified as the principle active component of turmeric that is responsible for many pharmacological properties [11] including anti-inflammatory, powerful antioxidant activity [12], and cancer-preventive properties [13]. Also, it lowers blood glucose and glycated 
hemoglobin levels by lowering oxidative stress in diabetic rats $[14,15]$. Several studies have reported that curcumin has hypolipemic action in rats [16]. It lowers serum cholesterol levels in diet induced hyperlipidemia [17] and triglycerides in alcohol induced toxicity [18]. It also increases cholesterol fecal excretion [19] and induces changes in gene expression involved in cholesterol homeostasis [20].

Prickly pear cactus (Opuntia ficus-indica) is a tree like cactus from the family Cactaceae [21]. The dried leaves of prickly pear cactus have been considered as one of the functional food by embracing essential ingredients, such as amino acids, taurine, carbohydrates, vitamin $\mathrm{C}$, minerals, and soluble fibers [22]. Prickly pear owns well-known antidiabetic and lipid lowering properties [23]. Also, it is very useful in obesity, alcohol-induced hangover, colitis, diarrhea, and benign prostatic hypertrophy (BPH) [24]. Many uses of cladodes (leaves) of cactus pear have been reported and utilized for their hypolipidemic properties [21, 24, 25]. Evidence showed that cactus pear reduces cholesterol levels in human and modify LDL composition [22].

Garlic (Allium sativum) from the family Alliacae is used as spice and medicinal herb [26]. It is used as raw, cooked, dried, and in form of tablets [27]. Garlichas protective actions against cardiovascular diseases. Numerous studies suggest that garlic can normalize serum lipids, enhances fibrinolytic activity, and reduces blood pressure [28].

The successful use of herbal combinations in treating or managing diseases or conditions over a single herb [29, 30] has led us to evaluate the antidyslipidemic properties of the combination of the above natural extracts versus each one alone in two different hyperlipidemic models [31]. Furthermore, in order to study the mechanism of action of each constituent, each product was tested in inhibiting the activity of HMG-CoA reductase enzyme. The long-term objective of this study, however, is enhancing the use of these natural extracts in lowering serum levels of cholesterol, LDL and triglycerides, and increasing serum HDL in order to reduce CVD and their adverse consequences.

\section{Materials and Methods}

2.1. Materials. Pluronic F-127, cholesterol and cholic, acid were obtained from Sigma-Aldrich, USA. Corn oil and Atorvastatin were supplied by the Jordanian Pharmaceutical Manufacturing Company (JPM). Artichoke leaves powdered extract $(>2.5 \%$ caffeolquinic acid) was purchased from Finzelberg, Germany; cactus dried leaves ( $>35 \%$ edible fibers) from Bioserae, France; common turmeric extract (Curcumins $>97.5 \%$ ) from Xi'an Tianxing Natural BioProducts Co Ltd, China, garlic powder extract (Alliin >3.4\%) from Flachsmann, Canada. The natural extracts were used as supplied and no attempts were carried to evaluate their compositions.

2.2. Animals and Treatments. Experiments were carried out on adult Spargue Dawley (SD) male rats weighing (200$250 \mathrm{~g}$ ). The animals were housed in an air-conditioned environment under a daily photoperiod $12 \mathrm{~h}$ light/12 h dark cycle and received standard chow and tap water ad libitum. Animals were kept for 2 weeks to allow acclimatization to the animal facility before starting the experiments. All experiments on animals were performed following the standards of European Community Council Directive of November 24, 1986 (86/609/EEC). The study protocol was revised and approved (9/2008) by the Ethical Committee of the Faculty of Pharmacy and Medical Sciences, University of Petra, Amman, Jordan.

2.3. Hyperlipidemia Induction Using Pluronic F-127. Pluronic F-127 is a nontoxic, hydrophilic nonionic surfactant that is administered parenterally in rodents to induce reversible hyperlipidemia [32-34]. Pluronic F-127 solution was prepared by dissolving the corresponding dose in cold normal saline and kept in the refrigerator overnight for complete dissolution. Twenty-four hours before the experiment, only water was offered to the rats. Treated groups ( $n=8 /$ time point) received a single intraperitoneal (i.p) injection of $300 \mathrm{mg} / \mathrm{kg}$ of Pluronic F-127. The controls were injected with normal saline only. During the $48 \mathrm{~h}$ of the experiment, blood samples at $0,4,12,24$, and $48 \mathrm{~h}$ were drawn. At each time point, rats were sacrificed under anesthesia and blood samples were directly collected from the heart. Blood samples were allowed to clot for 15 minutes and then centrifuged. Serum samples were kept frozen at $-20^{\circ} \mathrm{C}$ until date of analysis.

2.4. Hyperlipidemia Induction Using High-Fat Diet. High-fat diet consisted of standard diet mixed with $10 \%$ corn oil, $1 \%$ cholesterol, and $0.2 \%$ cholic acid. For this method of induction, rats were divided in two groups; group 1: control, fed with the normolipidemic diet, and group 2: fed with the cholesterol based formula. All groups had free access to water. After 10 days of feeding, fasting rats were sacrificed under anesthesia. Blood samples were taken directly from the heart and processed as previously described.

2.5. Antihyperlipidemic Effect of the Natural Extracts. To investigate the effect of the natural extracts to prevent the elevation of blood lipids, animals were divided into 8 groups with eight rats in each group. Each group received daily either water, atorvastatin $10 \mathrm{mg} / \mathrm{kg}$, artichoke leaves extract $26 \mathrm{mg} / \mathrm{kg}$, turmeric $80 \mathrm{mg} / \mathrm{kg}$, prickly pear leaves (PPL) $22 \mathrm{mg} / \mathrm{kg}$, garlic $17 \mathrm{mg} / \mathrm{kg}$, combination 1 (artichoke, turmeric, and PPL), or combination 2 (artichoke, turmeric, PPL, and garlic). These doses correspond to the human recommended daily doses of $1.8,5.6,1.5$, and $1.2 \mathrm{~g}$ /day for artichoke, turmeric, PPL, and garlic, respectively [6, $18,35,36]$. At the beginning of each experiment, rats were treated orally using an intragastric tube and they had free access to water and normal diet. In Pluronic F-127 induced hyperlipidemia, rats were divided into 9 groups $(n=$ 6/group). Group 1 served as negative control. For 2 weeks, rats were orally treated with the previously described doses and had free access to water and normal diet. Twenty-four hours before the last sample treatment, hyperlipidemia was induced using a single i.p injection of $300 \mathrm{mg} / \mathrm{kg}$ Pluronic 
F-127 after $24 \mathrm{~h}$ fasting. Group 1 was injected with normal saline only. At the end of the experiment, blood samples were collected from the hearts, centrifuged and kept at $-20^{\circ} \mathrm{C}$ until analysis. In High-Fat diet modeland after 14 days of treatment, rats were switched to be fed with High-Fat diet ( $10 \%$ corn oil, $1 \%$ cholesterol, and $0.2 \%$ cholic acid) along with the treatment. At the last day of the study, all animals were kept fasting (except for water) for $24 \mathrm{~h}$ before blood samples were collected directly from the heart and processed as previously described.

2.6. Biochemical Assay of Serum Lipids. Serum total cholesterol, triglycerides, and LDL were determined by enzymatic method using Analyticon Biotechnologies AG, Germany kits, while HDL was measured by using Labkit, Chemelex, S.A. The absorbance was measured at $546 \mathrm{~nm}$ wavelength using RA-50 Chemistry Analyzer, Technicon, USA.

2.7. HMG-CoA Reductase Activity Assay. The effect of the natural extracts on the activity of HMG-CoA reductase was assayed using an enzymatic assay kit from Sigma-Aldrich, USA.

2.8. Statistical Analysis. Results are expressed as mean \pm S.E.M (standard error of the mean). Data obtained were analyzed by ANOVA followed by Dunnett as post hoc test using SPSS program, version 17. $P$ values less than 0.05 were considered significant.

\section{Results}

3.1. Pluronic F-127 versus High-Fat Diet. Administration of Pluronic F-127 led to very high serum levels of triglycerides, cholesterol, and LDL, but not HDL in rats (Figure 1). After $4 \mathrm{~h}$, the increase in cholesterol and LDL was not significant as it is compared to the control, while triglycerides level increased significantly $(P<0.05)$. The increase in serum lipid levels continued till it reached a peak level at $24 \mathrm{~h}$ and plateaued for $48 \mathrm{~h}$ following the injection $(P<0.0001)$. However, the elevation in triglyceride level was much higher than cholesterol and LDL (Figure 1). Giving High-Fat diet to rats for 10 days, on the other hand, resulted in a significant increase in serum cholesterol, LDL, LDL/HDL ratio, and triglycerides $(P<0.001,0.001,0.001$, and 0.05 , resp.) (Table 1 ).

3.2. Antihyperlipidemic Effect of the Natural Extracts in Pluronic F-127 Induced Hyperlipidemia. A two-week treatment of PPL and garlic extracts prior to a single i.p injection of $300 \mathrm{mg} / \mathrm{kg}$ Pluronic F-127 resulted in decreasing significantly serum LDL levels $(P<0.05)$ (Table 2$)$. However, turmeric, PPL, and both combinations resulted in decreasing LDL/HDL ratios significantly $(P<0.05,0.001)$. Atorvastatin, on the other hand, did not cause any significant effect on serum lipid levels induced by Pluronic administration (Table 2).
TABLE 1: Effects of high-cholesterol diet on the serum lipid profile of rats.

\begin{tabular}{lcc}
\hline Parameter $(\mathrm{mg} / \mathrm{dL})$ & $\begin{array}{c}\text { Control }^{*} \\
n=6\end{array}$ & $\begin{array}{c}\text { High-cholesterol diet* } \\
n=6\end{array}$ \\
\hline Total cholesterol & $61 \pm 5$ & $94 \pm 3^{\mathrm{a}}$ \\
Triglyceride & $72 \pm 5$ & $92 \pm 4^{\mathrm{b}}$ \\
HDL & $19 \pm 2$ & $15 \pm 2$ \\
LDL & $52 \pm 4$ & $82 \pm 2^{\mathrm{a}}$ \\
LDL/HDL & $2.9 \pm 0.3$ & $6.2 \pm 0.7^{\mathrm{a}}$ \\
\hline
\end{tabular}

Data presented as means \pm SEM (standard error of the mean).

* Animals were fed high-cholesterol diet for 10 days.

Control animals received normal diet.

${ }^{\mathrm{a}} P<0.001$ and ${ }^{\mathrm{b}} P<0.05$ versus control.

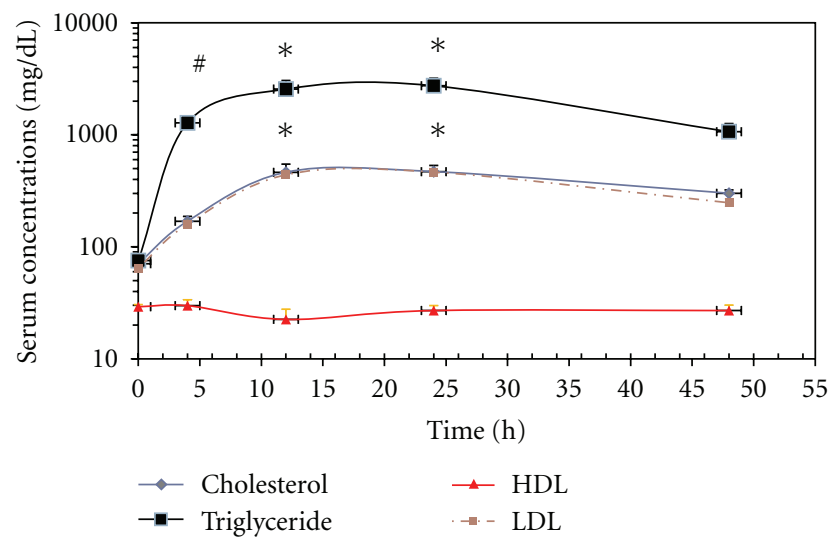

FIgURE 1: Changes in serum lipid levels at different times following a single i.p injection of $300 \mathrm{mg} / \mathrm{kg}$ Pluronic F-127. Values are presented as means \pm SEM, with $n=8$ for each data point. Symbols mean statistically significant; ${ }^{\#} P<0.05,{ }^{*} P<0.0001$, compared to control. Significant values for cholesterol are the same for LDL.

3.3. Antihyperlipidemic Effect of the Natural Extracts and Their Combinations in High-Fat Diet-Induced Hyperlipidemia. Treating the rats for 2 weeks either with a single product or with different combinations and then giving them High-Fat diet for only 10 days (with the treatment) gave the following results: none of the single product changed the level of any serum lipid profiles; the first combination (artichoke, turmeric and PPL) and the second one (artichoke, turmeric, PPL and garlic) lowered cholesterol and LDL levels by 8$12 \%$; decreased significantly serum triglycerides, LDL/HDL ratio, and increased significantly HDL levels $(P<0.0001)$ (Figure 2). On the other hand, Atorvastatin decreased significantly serum cholesterol, LDL levels $(P<0.05)$, LDL/HDL ratio $(P<0.0001)$, triglycerides $(P<0.0001)$, and increased significantly HDL levels $(P<0.0001)$.

However, keeping the rats under High-Fat diet for another 25 days (i.e., 5 weeks in total) resulted in a drop of serum cholesterol level in the control group that abolishes the significance effect of Atorvastatin on cholesterol level. However, all the natural extracts, except for PPL, and Atorvastatin resulted in a significant decrease in triglycerides levels $(P<0.01-0.0001)$ (Table 3$)$. The decrease in LDL level was also significant for all groups $(P<0.05-0.01)$ except 
TABLE 2: The effect of the natural extracts and their combinations on serum lipid levels in Pluronic F-127 induced hyperlipidemia.

\begin{tabular}{|c|c|c|c|c|c|}
\hline \multirow{2}{*}{ Groups } & \multicolumn{5}{|c|}{ Serum Lipid Parameters (mg/dL) } \\
\hline & Cholesterol & Triglycerides & HDL & LDL & LDL/HDL \\
\hline Normal & $75 \pm 2$ & $64 \pm 6$ & $39 \pm 5$ & $65 \pm 1$ & $1.9 \pm 0.2$ \\
\hline Control Pl. & $455 \pm 30$ & $2894 \pm 206$ & $33 \pm 3$ & $378 \pm 23$ & $11.5 \pm 0.7$ \\
\hline Atorvastatin & $460 \pm 14$ & $3021 \pm 176$ & $26 \pm 2$ & $377 \pm 13$ & $14.7 \pm 1.1$ \\
\hline Artichoke & $523 \pm 44$ & $3504 \pm 160$ & $41 \pm 8$ & $415 \pm 39$ & $11.7 \pm 2.3$ \\
\hline Turmeric & $487 \pm 20$ & $3365 \pm 153$ & $43 \pm 2$ & $387 \pm 18$ & $9.1 \pm 0.8^{\mathrm{a}}$ \\
\hline PPL & $388 \pm 17$ & $2888 \pm 190$ & $50 \pm 3$ & $308 \pm 15^{\mathrm{a}}$ & $6.2 \pm 0.5^{b}$ \\
\hline Garlic & $389 \pm 17$ & $3020 \pm 198$ & $25 \pm 2$ & $313 \pm 14^{\mathrm{a}}$ & $12.9 \pm 1.4$ \\
\hline Comb. 1 & $573 \pm 28$ & $3723 \pm 159$ & $57 \pm 4$ & $472 \pm 24$ & $8.3 \pm 0.4^{\mathrm{a}}$ \\
\hline Comb. 2 & $510 \pm 37$ & $3253 \pm 204$ & $54 \pm 2$ & $413 \pm 33$ & $7.7 \pm 0.7^{\mathrm{a}}$ \\
\hline
\end{tabular}

Data presented as means \pm SEM with 6 rats in each group. Control Pl.: control group given only Pluronic F-127. PPL: prickly pear leaves. Comb. 1: combination 1 (artichoke, turmeric, and PPL). Comb. 2: combination 2 (artichoke, turmeric, PPL, and garlic). Statistical analysis was done using ANOVA, ${ }^{a} P<0.05$, and ${ }^{\mathrm{b}} \mathrm{P}<0.0001$ compared to control Pl.

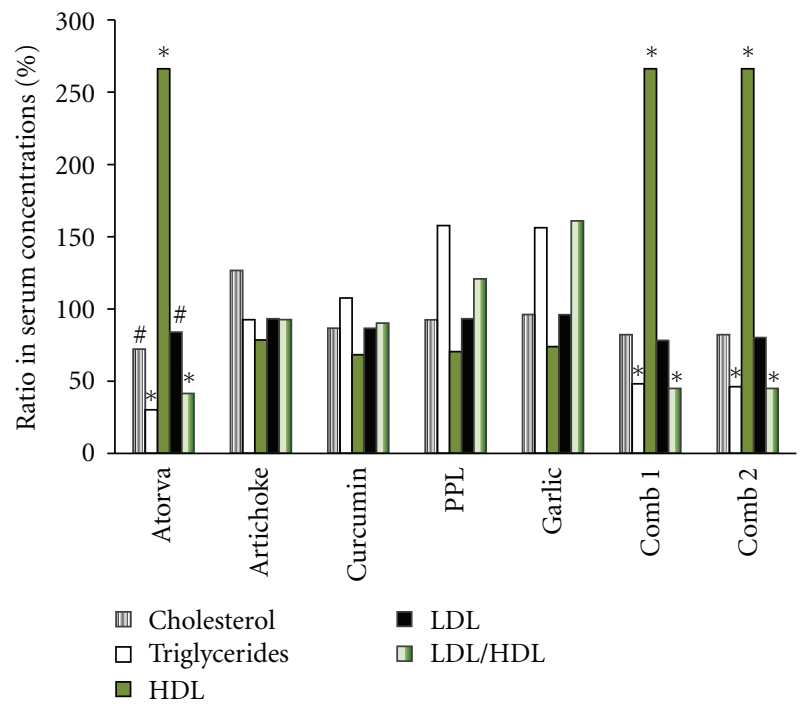

FIgURE 2: The effect of the two combinations on serum lipid levels in comparison with the single treatment. Atorva: atorvastatin $10 \mathrm{mg} / \mathrm{kg}$. PPL: prickly pear leaves. Comb. 1: combination 1 (artichoke, turmeric, and PPL). Comb. 2: combination 2 (artichoke, turmeric, PPL, and garlic). Values are presented as means \pm SEM, with $n=8$ for each data point. Symbols mean statistically significant; ${ }^{\#} P<0.05$ and ${ }^{*} P<0.0001$, compared to the control. For clarity reasons, error bars were not applied to the figure given that error range was $2-5 \%$.

for Atorvastatin. Treatment with turmeric and PPL increased HDL levels significantly $(P<0.01)$, (Table 3$)$. Moreover, all the treatments decreased significantly LDL/HDL ratio $(P<$ 0.05-0.0001).

3.4. Effect of the Natural Extracts on HMG-CoA Reductase Activity. The effect of the natural extracts on the activity of HMG-CoA reductase is given in Figure 3. Using three different concentrations for each product and for Atorvastatin, as a positive control, resulted in that Atorvastatin's three concentrations significantly inhibited the activity of the enzyme

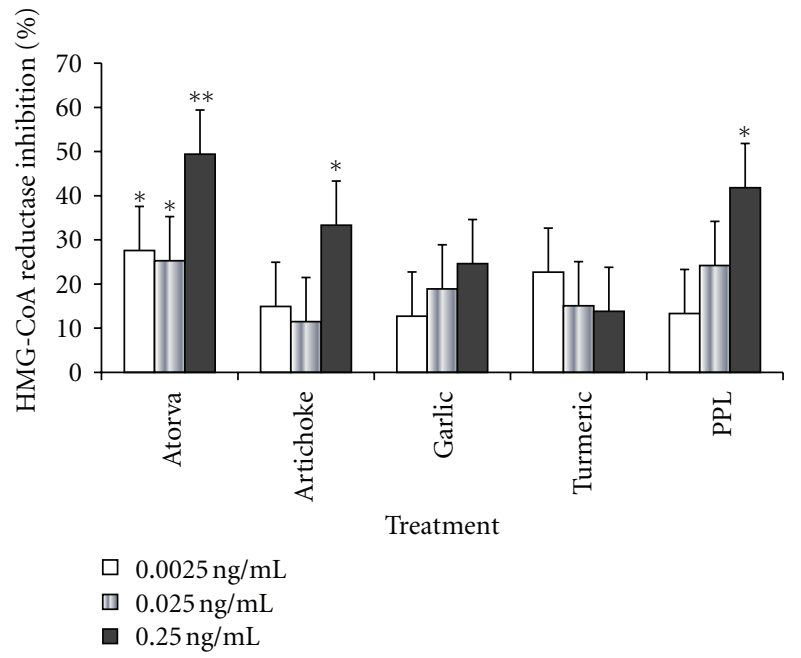

FIGURE 3: The effect of the natural extracts on HMG-CoA reductase activity at different concentrations. Atorva: Atorvastatin; PPL: prickly pear leaves. Values are presented as means \pm S.D. Symbols mean statistically significant; ${ }^{*} P<0.05$ and ${ }^{* *} P<0.01$, compared to the negative control.

$(P<0.05-0.01)$. Artichoke's and PPL's highest concentrations also inhibited the enzyme's activity significantly $(P<$ $0.05)$. Although garlic did not have a significant effect on the activity of the enzyme, it is obvious that its inhibitory effect is concentration dependent. On the other hand, turmeric did not have a significant effect on the enzyme's activity, at the used concentrations.

\section{Discussion}

In order to understand the relationship between disorders in cholesterol metabolism and atherogenesis and to test possible treatments for the reduction of serum lipid levels, various models are found to induce hyperlipidemia in rats $[2,37]$. As intrinsic inducer, the surfactant Pluronic produces massive but reversible hyperlipidemia in rodents 
TABLE 3: The effect of the natural extracts, separately, on serum lipid levels after 7-week period of treatment that included 5 weeks feeding high-fat diet.

\begin{tabular}{|c|c|c|c|c|c|}
\hline \multirow{2}{*}{ Groups } & \multicolumn{5}{|c|}{ Serum lipid parameters $(\mathrm{mg} / \mathrm{dL})$} \\
\hline & Cholesterol & Triglycerides & HDL & LDL & LDL/HDL \\
\hline Control & $85 \pm 3$ & $65 \pm 7$ & $16 \pm 2$ & $75 \pm 2$ & $5.2 \pm 0.5$ \\
\hline Atorvastatin & $86 \pm 3$ & $44 \pm 2^{\mathrm{b}}$ & $24 \pm 3$ & $76 \pm 3$ & $3.5 \pm 0.5^{\mathrm{a}}$ \\
\hline Artichoke & $79 \pm 3$ & $43 \pm 3^{\mathrm{b}}$ & $21 \pm 3$ & $64 \pm 3^{a}$ & $3.5 \pm 0.5^{\mathrm{a}}$ \\
\hline Turmeric & $75 \pm 3$ & $27 \pm 1^{\mathrm{c}}$ & $29 \pm 4^{\mathrm{b}}$ & $61 \pm 2^{\mathrm{b}}$ & $2.5 \pm 0.4^{\mathrm{c}}$ \\
\hline PPL & $72 \pm 2^{b}$ & $49 \pm 1$ & $28 \pm 2^{b}$ & $60 \pm 3^{\mathrm{b}}$ & $2.2 \pm 0.1^{\mathrm{c}}$ \\
\hline Garlic & $77 \pm 3$ & $35 \pm 4^{\mathrm{c}}$ & $24 \pm 2$ & $62 \pm 3^{b}$ & $2.7 \pm 0.2^{\mathrm{b}}$ \\
\hline
\end{tabular}

Data presented as means \pm SEM of 8 rats in each group. PPL: prickly pear leaves. Statistical analysis was done using ANOVA; ${ }^{\mathrm{a} P}<0.05,{ }^{\mathrm{b}} P \leq 0.01,{ }^{\mathrm{c}} P<0.0001$, compared to the control group.

after acute administration [34, 38]. This rise in circulating lipoproteins levels is caused by modifications in a number of biochemical pathways that is involved in lipoprotein metabolism [34], mainly an increase in serum triglycerides due to the direct inhibition of lipoprotein lipase which is the enzyme responsible for triglycerides degradation $[31,33]$. The increase in serum cholesterol is potentially due to indirect activation of HMG-CoA reductase that is responsible for cholesterol de novo synthesis [31, 39]. Using this extreme model of hyperlipidemia induction, PPL, and garlic decreased significantly serum LDL levels whereas the combination 1 and 2 resulted in decreasing LDL/HDL ratio as well as PPL and turmeric extracts. Preadministration of Atorvastatin, on the other hand, did not prevent the changes induced by Pluronic administration indicating that the increase in serum cholesterol in such model may not be due to indirect activating HMG-CoA reductase and/or the latter has a minor role.

As extrinsic inducer, 10-day high-fat diet caused a significant increase in serum cholesterol and LDL, triglycerides levels and decreased HDL. Pretreating the rats for 14 days with any of the single products did not prevent or decrease serum lipid profiles. On the other hand, the combinations artichoke, turmeric and PPL or artichoke, turmeric, PPL, and garlic decreased significantly serum triglycerides level, and LDL/HDL ratio; increased significantly HDL, and also mildly lowered cholesterol and LDL levels by $8-12 \%$, respectively. Thus, such natural extracts combination for long-term use would give more significant normalization of dyslipidemia.

When the High-Fat diet continued for longer period (5 weeks), it did not increase the total serum cholesterol level but increased serum LDL and decreased HDL levels. It has been noticed that after 7-10 days of High-Fat diet feeding, serum cholesterol, triglyceride, and LDL levels increased significantly in comparison to baseline levels. During the next 4 weeks of High-Fat diet feeding to rats, it was noticed that the level of serum cholesterol and triglycerides started to decrease in the control group as well as the treated groups but the ratio of LDL/HDL stayed significantly high. This could be explained by that long-term High-Fat diet induces saturation in cholesterol synthesis inside hepatocytes, and thus LDL receptor expression is reduced justifying high LDL serum concentrations [37, 40]. In addition, long-term treatment of plant sterol with cholesterol might reduce intestinal cholesterol absorption $[41,42]$ but this would be noticed after long-term treatment [43].

Englisch et al. (2000) have reported that the artichoke extract significantly lowered cholesterol and LDL (18.5\% and $22.9 \%$, resp.), in a randomized double-blinded placebocontrol trial in which 143 hypercholesterolemic patients received either $1800 \mathrm{mg} /$ day of the extracts or matched placebo for six weeks $[6,8]$. In this study, artichoke leaves extracts significantly lowered serum triglycerides, LDL, and LDL/HDL ratio. These modulations are attributed partially to inhibiting HMG-CoA reductase as seen herein and by others [5].

We have shown herein that turmeric decreased significantly serum triglyceride, LDL, LDL/HDL ratio, and increased serum HDL level. Although turmeric effect on serum cholesterol was not significant, it decreased its level by $12 \%$. The hypocholesterolemic effect of turmeric has been widely investigated. In rats fed high-cholesterol diet for 7 days, Arafa (2005) investigated the hypolipidemic effect of curcumin $(0.5 \% \mathrm{w} / \mathrm{w})$ when added to diet and reported that curcumin decreased cholesterol by $21 \%$ and LDL by $42.5 \%$, while HDL level was increased by 50\% [17]. Another report has shown that curcumin decreased total cholesterol in serum by $12 \%$ and increased HDL by $29 \%$ in human subjects after oral administration of $0.5 \mathrm{~g} /$ day of curcumin [44]. In healthy human volunteers receiving $500 \mathrm{mg}$ /day curcumin for 7 days, serum cholesterol was lowered by $11.63 \%$ and HDL level was increased by $29 \%$ [45]. The mechanism by which curcumin affects lipid levels is not related to HMG-CoA inhibition but could be due to an increase in the activity of hepatic cholesterol-7a-hydoxylase thereby enhancing cholesterol catabolism [12]. Other mechanisms of turmeric may include increasing cholesterol fecal excretion [19], inducing changes in gene expression involved in cholesterol homeostasis [20] and through a local effect on cholesterol absorption [10].

Prickly pear dried leaves significantly decreased serum cholesterol, LDL levels, and LDL/HDL ratio and significantly increased serum HDL levels. Although its effect on triglycerides level was not significant, PPL decreased serum triglycerides by $23.5 \%$. Many authors mentioned that the cladodes of Opuntia ficus indica are utilized for their hypolipidemic properties $[21,24,25]$. Evidence showed that cactus pear reduces cholesterol levels in human and 
modify LDL composition [22]. Prickly pear lowers LDL levels most likely as a consequence of its pectin content [23]. Rodriguez-Fragoso also clarified that pectin is able to alter hepatic cholesterol metabolism without affecting cholesterol absorption [24]. PPL at a dose of $1.6 \mathrm{~g} /$ meal given to 42 females above 45 years increased serum HDL levels and decreased triglycerides and LDL levels [35]. In the current study, PPL significantly inhibited the activity of HMG-CoA reductase and was concentration dependent.

Various extracts of garlic have been shown to lower serum cholesterol, triglycerides, and LDL in rodents and humans $[26,46]$. Chetty studied the effect of $2 \%$ dietary garlic given to rats fed $1 \%$ cholesterol with olive oil. The results indicated a significant decrease in total serum cholesterol and LDL with an increase in HDL levels [41]. Many authors stated that garlic exert its antihyperlipidemic effect by inhibiting HMG-CoA reductase and thus inhibit hepatic cholesterol biosynthesis [28, 36, 47]. In our study, although garlic did not have a significant effect on the activity of the enzyme, it is obvious that its inhibitory effect is concentration dependent (Figure 3). Other mechanisms include inhibiting intestinal cholesterol absorption and enhancing cholesterol turnover to bile acids $[36,48]$.

Since treating or managing dyslipidemia requires a lifelong use of antihyperlipidemic drugs, it is necessary to use a combination of natural extracts that exert a similar outcome with multi-mechanism of action and have less adverse events. Such combination can be used alone as prophylactic, a booster to a known antihyperlipidemic drugs, or as lifelong phytopharmaceuticals. As presented herein, neither of the artichoke leaves extract, turmeric extract, prickly pear dried leaves, and garlic extract showed any effect on shortterm treatment in High-Fat diet model but the combination effect to normalize dyslipidemia is additive. Thus using the combination of artichoke leaves extract, turmeric extract, prickly pear dried leaves, and garlic extract for short-term, as well as long-term, treatment can prevent dyslipidemia; partially through inhibiting HMG-CoA reductase.

\section{Conflict of Interests}

The authors have declared that there is no conflict of interests.

\section{Acknowledgments}

This work was supported by a grant from the Deanship of Scientific Research at Petra University, Amman, Jordan and by Delass Natural Products, Naor, Jordan.

\section{References}

[1] H. Harnafi, H. Serghini Caid, N. el Houda Bouanani, M. Aziz, and S. Amrani, "Hypolipemic activity of polyphenolrich extracts from Ocimum basilicum in Triton WR-1339induced hyperlipidemic mice," Food Chemistry, vol. 108, no. 1, pp. 205-212, 2008.

[2] S. Bolkent, R. Yanardag, O. Karabulut-Bulan, and B. Yesilyaprak, "Protective role of Melissa officinalis L. extract on liver of hyperlipidemic rats: a morphological and biochemical study," Journal of Ethnopharmacology, vol. 99, no. 3, pp. 391398, 2005.

[3] V. Lattanzio, P. A. Kroon, V. Linsalata, and A. Cardinali, "Globe artichoke: a functional food and source of nutraceutical ingredients," Journal of Functional Foods, vol. 1, no. 2, pp. 131-144, 2009.

[4] G. Lupattelli, S. Marchesi, R. Lombardini et al., "Artichoke juice improves endothelial function in hyperlipemia," Life Sciences, vol. 76, no. 7, pp. 775-782, 2004.

[5] T. Saénz Rodriguez, D. García Giménez, and R. de la Puerta Vázquez, "Choleretic activity and biliary elimination of lipids and bile acids induced by an artichoke leaf extract in rats," Phytomedicine, vol. 9, no. 8, pp. 687-693, 2002.

[6] W. Englisch, C. Beckers, M. Unkauf, M. Ruepp, and V. Zinserling, "Efficacy of artichoke dry extract in patients with hyperlipoproteinemia," Arzneimittel-Forschung, vol. 50, no. 3, pp. 260-265, 2000.

[7] M. H. Pittler, C. O. Thompson, and E. Ernst, "Artichoke leaf extract for treating hypercholesterolaemia," Cochrane Database of Systematic Reviews, no. 3, Article ID CD003335, 2002.

[8] R. Bundy, A. F. Walker, R. W. Middleton, C. Wallis, and H. C. R. Simpson, "Artichoke leaf extract (Cynara scolymus) reduces plasma cholesterol in otherwise healthy hypercholesterolemic adults: a randomized, double blind placebo controlled trial," Phytomedicine, vol. 15, no. 9, pp. 668-675, 2008.

[9] P. Anand, S. G. Thomas, A. B. Kunnumakkara et al., "Biological activities of curcumin and its analogues (Congeners) made by man and mother nature," Biochemical Pharmacology, vol. 76, no. 11, pp. 1590-1611, 2008.

[10] E. M. Jang, M. S. Choi, U. J. Jung et al., "Beneficial effects of curcumin on hyperlipidemia and insulin resistance in highfat-fed hamsters," Metabolism, vol. 57, no. 11, pp. 1576-1583, 2008.

[11] A. Goel, A. B. Kunnumakkara, and B. B. Aggarwal, "Curcumin as "curecumin": from kitchen to clinic," Biochemical Pharmacology, vol. 75, no. 4, pp. 787-809, 2008.

[12] M. C. Houston, S. Fazio, F. H. Chilton et al., "Nonpharmacologic treatment of dyslipidemia," Progress in Cardiovascular Diseases, vol. 52, no. 2, pp. 61-94, 2009.

[13] A. Kuhad and K. Chopra, "Curcumin attenuates diabetic encephalopathy in rats: behavioral and biochemical evidences," European Journal of Pharmacology, vol. 576, no. 1-3, pp. 34-42, 2007.

[14] B. B. Aggarwal and K. B. Harikumar, "Potential therapeutic effects of curcumin, the anti-inflammatory agent, against neurodegenerative, cardiovascular, pulmonary, metabolic, autoimmune and neoplastic diseases," International Journal of Biochemistry and Cell Biology, vol. 41, no. 1, pp. 40-59, 2009.

[15] K. Karthikesan, L. Pari, and V. P. Menon, "Protective effect of tetrahydrocurcumin and chlorogenic acid against streptozotocin-nicotinamide generated oxidative stress induced diabetes," Journal of Functional Foods, vol. 2, no. 2, pp. 134-142, 2010.

[16] Q. Kang and A. Chen, "Curcumin suppresses expression of low-density lipoprotein (LDL) receptor, leading to the inhibition of LDL-induced activation of hepatic stellate cells," British Journal of Pharmacology, vol. 157, no. 8, pp. 1354-1367, 2009.

[17] H. M. M. Arafa, "Curcumin attenuates diet-induced hypercholesterolemia in rats," Medical Science Monitor, vol. 11, no. 7, pp. BR228-BR234, 2005. 
[18] R. Rukkumani, M. Sri Balasubashini, P. Vishwanathan, and V. P. Menon, "Comparative effects of curcumin and photoirradiated curcumin on alcohol- and polyunsaturated fatty acid-induced hyperlipidemia," Pharmacological Research, vol. 46, no. 3, pp. 257-264, 2002.

[19] D. S. Rao, N. C. Sekhara, M. N. Satyanarayana, and M. Srinivasan, "Effect of curcumin on serum and liver cholesterol levels in the rat," Journal of Nutrition, vol. 100, no. 11, pp. 1307-1315, 1970.

[20] D. Peschel, R. Koerting, and N. Nass, "Curcumin induces changes in expression of genes involved in cholesterol homeostasis," Journal of Nutritional Biochemistry, vol. 18, no. 2, pp. 113-119, 2007.

[21] M. de Leo, M. B. D. Abreu, A. M. Pawlowska, P. L. Cioni, and A. Braca, "Profiling the chemical content of Opuntia ficus-indica flowers by HPLC-PDA-ESI-MS and GC/EIMS analyses," Phytochemistry Letters, vol. 3, no. 1, pp. 48-52, 2010.

[22] M. F. Ramadan and J. T. Mörsel, "Oil cactus pear (Opuntia ficus-indica L.)," Food Chemistry, vol. 82, no. 3, pp. 339-345, 2003.

[23] R. Wolfram, A. Budinsky, Y. Efthimiou, J. Stomatopoulos, A. Oguogho, and H. Sinzinger, "Daily prickly pear consumption improves platelet function," Prostaglandins Leukotrienes and Essential Fatty Acids, vol. 69, no. 1, pp. 61-66, 2003.

[24] L. Rodriguez-Fragoso, J. Reyes-Esparza, S. W. Burchiel, D. Herrera-Ruiz, and E. Torres, "Risks and benefits of commonly used herbal medicines in Mexico," Toxicology and Applied Pharmacology, vol. 227, no. 1, pp. 125-135, 2008.

[25] M. Ennouri, H. Fetoui, E. Bourret, N. Zeghal, and H. Attia, "Evaluation of some biological parameters of Opuntia ficus indica. 1. Influence of a seed oil supplemented diet on rats," Bioresource Technology, vol. 97, no. 12, pp. 1382-1386, 2006.

[26] K. Rahman, "Garlic and aging: new insights into an old remedy," Ageing Research Reviews, vol. 2, no. 1, pp. 39-56, 2003.

[27] V. Keshetty, R. G. SrinivasPabba, J. M. Kandukuri, and V. Allenki, "Antihyperlipidemic activity of methanolic extract of garlic (Allium sativum L.) in triton X-100 induced hyperlipidemic rats," Journal of Pharmacy Research, vol. 2, no. 5, pp. 777-780, 2009.

[28] M. P. McRae, "A review of studies of garlic (Allium sativum) on serum lipids and blood pressure before and after 1994: does the amount of allicin released from garlic powder tablets play a role?" Journal of Chiropractic Medicine, vol. 4, no. 4, pp. 182190, 2005.

[29] H. Wagner and G. Ulrich-Merzenich, "Synergy research: approaching a new generation of phytopharmaceuticals," Phytomedicine, vol. 16, no. 2-3, pp. 97-110, 2009.

[30] N. Qinna, H. Taha, K. Z. Matalka, and A. A. Badwan, "A new herbal combination, etana, for enhancing erectile function: an efficacy and safety study in animals," International Journal of Impotence Research, vol. 21, no. 5, pp. 315-320, 2009.

[31] H. J. Jung, J. H. Nam, H. J. Park et al., "The MeOH extract of Pleurospermum kamtschaticum and its active component buddlejasaponin (IV) inhibits intrinsic and extrinsic hyperlipidemia and hypercholesterolemia in the rat," Journal of Ethnopharmacology, vol. 112, no. 2, pp. 255-261, 2007.

[32] T. P. Johnston and W. K. Palmer, "Effect of poloxamer 407 on the activity of microsomal 3-hydroxy-3- methylglutaryl CoA reductase in rats," Journal of Cardiovascular Pharmacology, vol. 29, no. 5, pp. 580-585, 1997.

[33] S. Megalli, F. Aktan, N. M. Davies, and B. D. Roufogalis, "Phytopreventive anti-hyperlipidemic effects of Gynostemma pentaphyllum in rats," Journal of Pharmacy and Pharmaceutical Sciences, vol. 8, no. 3, pp. 507-515, 2005.

[34] A. Shayeganpour, H. Korashy, J. P. Patel, A. O. S. El-Kadi, and D. R. Brocks, "The impact of experimental hyperlipidemia on the distribution and metabolism of amiodarone in rat," International Journal of Pharmaceutics, vol. 361, no. 1-2, pp. 78-86, 2008.

[35] E. Linarès, C. Thimonier, and M. Degre, "The effect of NeOpuntia on blood lipid parameters-risk factors for the metabolic syndrome (Syndrome X)," Advances in Therapy, vol. 24, no. 5, pp. 1115-1125, 2007.

[36] C. Ulbricht, E. Basch, S. Basch, and J. K. Bryan, "An evidencebased review of garlic and its hypolipidemic properties by the natural standard research collaboration scientific evidence for common/studied uses," Medicine, vol. 2, pp. 1-7, 2010.

[37] S. L. Matos, H. de Paula, M. L. Pedrosa et al., "Dietary models for inducing hypercholesterolemia in rats," Brazilian Archives of Biology and Technology, vol. 48, no. 2, pp. 203-209, 2005.

[38] V. C. Cogger, S. N. Hilmer, D. Sullivan, M. Muller, R. Fraser, and D. G. Le Couteur, "Hyperlipidemia and surfactants: the liver sieve is a link," Atherosclerosis, vol. 189, no. 2, pp. 273281, 2006.

[39] T. P. Johnston and W. K. Palmer, "Mechanism of poloxamer 407-induced hypertriglyceridemia in the rat," Biochemical Pharmacology, vol. 46, no. 6, pp. 1037-1042, 1993.

[40] J. M. Berg, J. L. Tymoczko, and L. Stryer, Biochemistry, W. H. Freeman, New York, NY, USA, 5th edition, 2002.

[41] K. N. Chetty, L. Calahan, K. C. Harris et al., "Garlic attenuates hypercholesterolemic risk factors in olive oil fed rats and high cholesterol fed rats," Pathophysiology, vol. 9, no. 3, pp. 127132, 2003.

[42] L. H. Ellegård, S. W. Andersson, A. L. Normén, and H. A. Andersson, "Dietary plant sterols and cholesterol metabolism," Nutrition Reviews, vol. 65, no. 1, pp. 39-45, 2007.

[43] G. García-Llatas and M. T. Rodríguez-Estrada, "Current and new insights on phytosterol oxides in plant sterol-enriched food," Chemistry and Physics of Lipids, vol. 164, no. 6, pp. 607624, 2011.

[44] L. Baum, S. K. K. Cheung, V. C. T. Mok et al., "Curcumin effects on blood lipid profile in a 6-month human study," Pharmacological Research, vol. 56, no. 6, pp. 509-514, 2007.

[45] K. B. Soni and R. Kuttan, "Effect of oral curcumin administration on serum peroxides and cholesterol levels in human volunteers," Indian Journal of Physiology and Pharmacology, vol. 36, no. 4, pp. 273-275, 1992.

[46] M. J. Budoff, N. Ahmadi, K. M. Gul et al., "Aged garlic extract supplemented with B vitamins, folic acid and 1arginine retards the progression of subclinical atherosclerosis: a randomized clinical trial," Preventive Medicine, vol. 49, no. 2-3, pp. 101-107, 2009.

[47] I. Durak, M. Kavutcu, B. Aytaç et al., "Effects of garlic extract consumption on blood lipid and oxidant/antioxidant parameters in humans with high blood cholesterol," Journal of Nutritional Biochemistry, vol. 15, no. 6, pp. 373-377, 2004.

[48] M. Corzo-Martinez, N. Corzo, and M. Villamiel, "Biological properties of onions and garlic," Trends in Food Science and Technology, vol. 18, no. 12, pp. 609-625, 2007. 

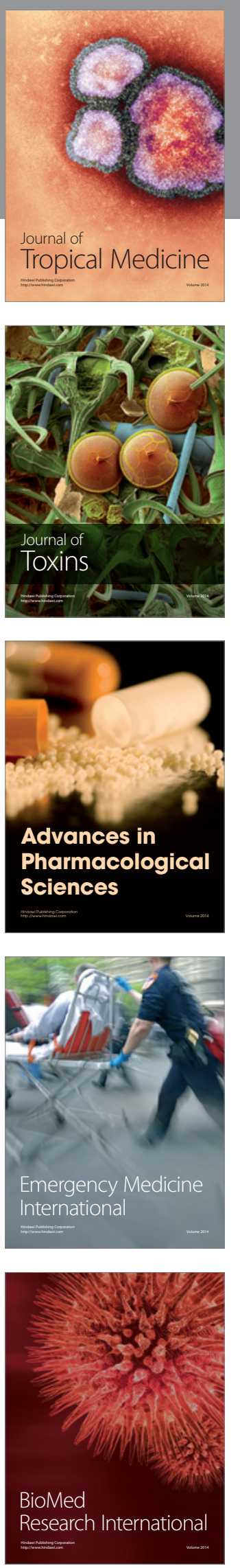
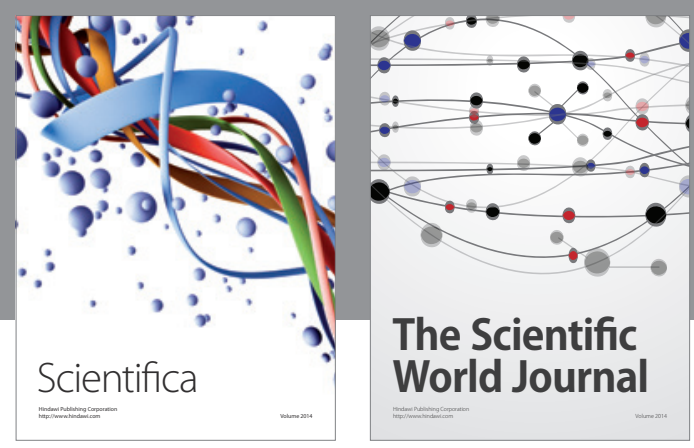

The Scientific World Journal
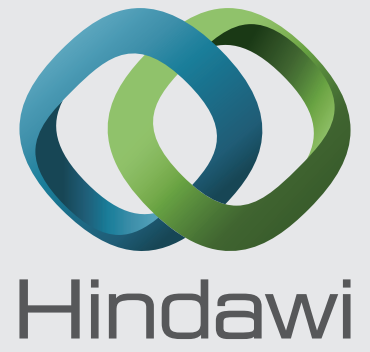

Submit your manuscripts at

http://www.hindawi.com
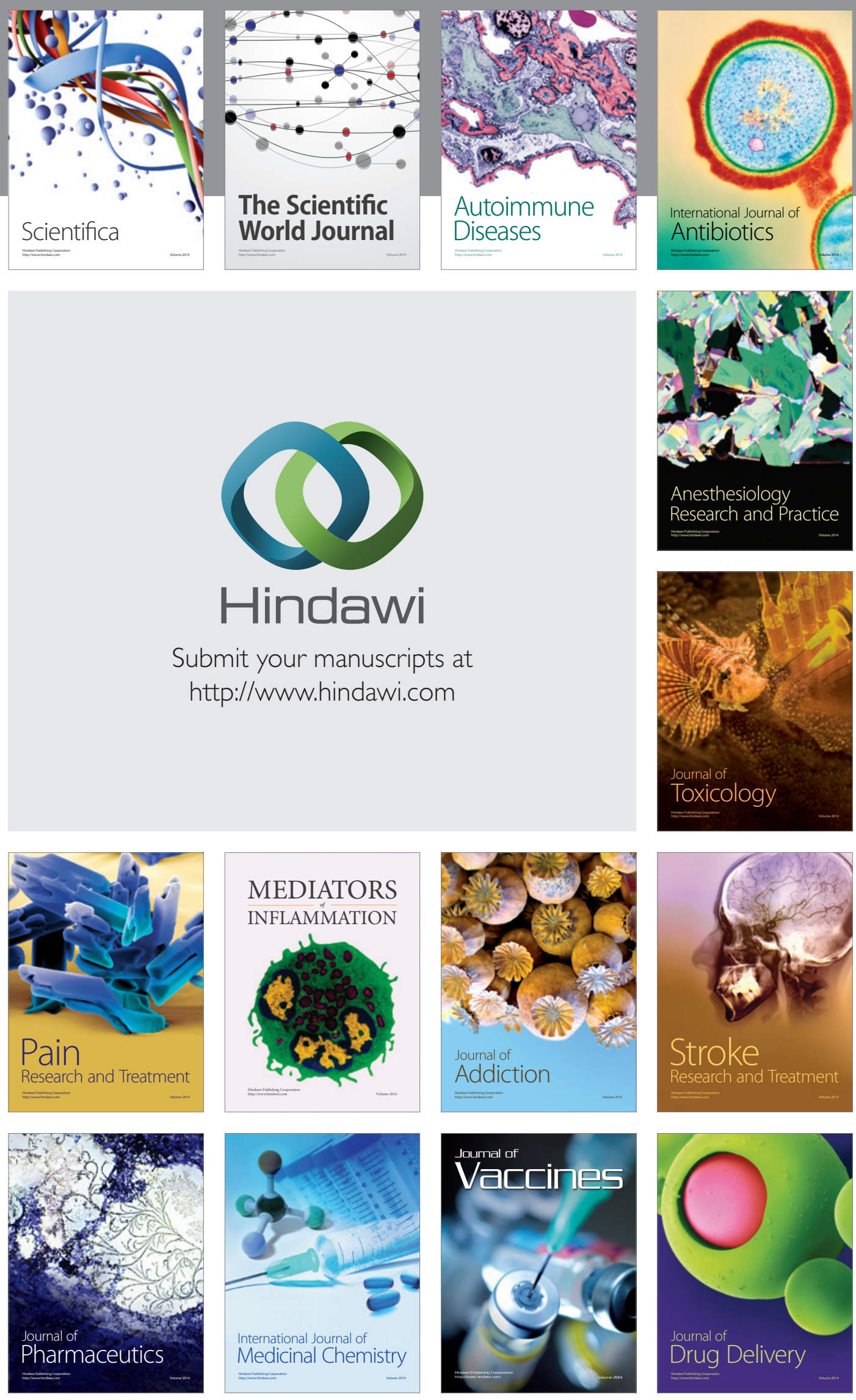